\title{
Hábitos de estudo de acadêmicos de fisioterapia em ensino remoto
}

\author{
Study habits of physical therapy students in remote teaching \\ Hábitos de estudio de los estudiantes de fisioterapia en la docencia a distancia
}

\section{Resumo}

No ano de 2020 houve a transferência massiva de estudantes que vinha acompanhando a faculdade de forma presencial para o ensino remoto, como solução a pandemia do COVID-19. Dessa maneira, fez-se necessário a reformulação da forma de ensinar, onde docentes tiveram que se capacitar para utilizar as tecnologias digitais adequadas para proporcionar o ensino remoto aos alunos, que por sua vez, também tiveram que se adaptar e buscar estratégias de estudo e aprendizagem. Assim, esse estudo objetivou analisar os hábitos de estudo de acadêmicos de fisioterapia em ensino remoto. Trata-se de uma pesquisa transversal, descritiva, com abordagem quantitativa, realizada com acadêmicos do curso de Fisioterapia de uma instituição de ensino superior privada. A coleta de dados ocorreu através de um questionário contendo questões objetivas, utilizando a plataforma Google Forms. Participaram do estudo 262 acadêmicos, sendo 76,72\% do sexo feminino, com idade média de 20,16 anos, pertencentes aos últimos anos do curso (54,58\%). A maior parcela relata nunca ter reprovado disciplinas (61,83\%), afirmam estudar remotamente entre uma e três horas por dia $(37,79 \%)$ e apresentaram-se com hábitos de estudo inadequados em todas as categorias investigadas. Ao analisar a autopercepção de rendimento nessa modalidade de ensino, o hábito inadequado é predominante em todas as percepções de rendimento acadêmico. O conhecimento do perfil discente, aliado às suas percepções acadêmicas e hábitos de estudo, podem conferir adaptações necessárias à continuidade do processo ensino-aprendizagem no ensino remoto.

Palavras-chave: Hábitos de estudo; Aprendizagem; Ensino remoto; Fisioterapia.

\section{Abstract}

In 2020 there was the massive transfer of students who had been following the college in person for remote education, as a solution to the COVID-19 pandemic. Thus, it was necessary to reformulate the way of teaching, where teachers had to be able to use the appropriate digital technologies to provide remote teaching to students, who in turn also had 
to adapt and seek study and learning strategies. Thus, this study aimed to analyze the study habits of physiotherapy students in remote teaching. This is a cross-sectional, descriptive, quantitative approach, conducted with physical therapy students from a private higher education institution. Data collection occurred through a questionnaire containing objective questions, using the Google Forms platform. The study included 262 students, $76.72 \%$ of them female, with a mean age of 20.16 years, belonging to the last years of the course $(54.58 \%)$. The largest proportion reported never having failed subjects $(61.83 \%)$, claiming to study remotely between one and three hours a day $(37.79 \%)$ and presented inadequate study habits in all categories investigated. When analyzing self-perception of income in this type of teaching, inadequate habit is predominant in all perceptions of academic performance. Knowledge of the student profile, together with their academic perceptions and study habits, can confer necessary adaptations to the continuity of the teaching-learning process in remote teaching.

Keywords: Study habits; Learning; Remote teaching; Physiotherapy.

\section{Resumen}

En 2020 se realizó el traslado masivo de estudiantes que habían estado siguiendo la universidad en persona para la educación remota, como solución a la pandemia de COVID-19. Por lo tanto, era necesario reformular la forma de enseñar, donde los maestros tenían que ser capaces de utilizar las tecnologías digitales apropiadas para proporcionar enseñanza remota a los estudiantes, quienes a su vez también tenían que adaptarse y buscar estrategias de estudio y aprendizaje. Así, este estudio tuvo como objetivo analizar los hábitos de estudio de los estudiantes de fisioterapia en la enseñanza a distancia. Este es un enfoque transversal, descriptivo, cuantitativo, realizado con estudiantes de fisioterapia de una institución privada de educación superior. La recolección de datos se produjo a través de un cuestionario que contenía preguntas objetivas, utilizando la plataforma Google Forms. El estudio incluyó a 262 estudiantes, el 76,72\% de ellos mujeres, con una edad media de 20,16 años, pertenecientes a los últimos años del curso $(54,58 \%)$. La mayor proporción reportó no haber fallado nunca a los sujetos $(61,83 \%)$, afirmando estudiar a distancia entre una y tres horas al día $(37,79 \%)$ y presentó hábitos de estudio inadecuados en todas las categorías investigadas. Al analizar la autopercepción de los ingresos en este tipo de enseñanza, el hábito inadecuado es predominante en todas las percepciones del rendimiento académico. El conocimiento del perfil del alumno, junto con sus percepciones académicas y hábitos de estudio, puede conferir las adaptaciones necesarias a la continuidad del proceso de enseñanza-aprendizaje en la docencia a distancia.

Palabras clave: Hábitos de estudio; Aprendizaje; Enseñanza a distancia; Fisioterapia.

\section{Introdução}

A educação sempre esteve em processo de mudança e no ano de 2020 não foi diferente, pois houve a transferência massiva de estudantes que vinham acompanhando a faculdade de forma presencial para o ensino remoto, como solução a pandemia do novo Coronavírus (COVID-19), que teve como medidas sanitárias, a restrição ao contato social (Garcia et al., 2020).

Em caráter emergencial, fez-se necessário a reformulação da forma de ensinar, onde os docentes tiveram que se capacitar para utilizar as tecnologias digitais adequadas da modalidade de ensino à distância para proporcionar o ensino remoto aos seus alunos, que por sua vez, também tiveram que se adaptar e buscar estratégias de estudo e aprendizagem para encarar essa nova realidade (Nunes \& Dutra, 2020).

Dentro desta perspectiva, sabe-se que situações emergenciais requerem a aplicação de medidas que tragam respostas eficazes, rápidas e que atendam demandas urgentes. No âmbito educacional, estas medidas assumem vertentes que apoiam tecnologias digitais e práticas inovadoras (Garcia et al., 2020). No entanto, algumas iniquidades podem surgir quanto ao acesso à educação neste momento, tanto para os discentes como para os docentes. A falta de infraestrutura, de acesso à internet e da capacidade para utilizar as tecnologias de informação e comunicação, podem fragilizar o processo de ensino e aprendizagem e interferir no envolvimento acadêmico (Cavalcante et al., 2020).

Nas últimas décadas, nota-se uma expansão significativa no ensino superior do Brasil com o aumento no número de instituições e de alunos matriculados. Mesmo com os infinitos desafios que o âmbito acadêmico acarreta, a busca pela formação no ensino superior é crescente. Porém, o desenvolvimento, aprendizagem e permanência do estudante nestas instituições são influenciados por diversas variáveis, tendo o envolvimento acadêmico como a principal (Fior \& Mercuri, 2018; Oliveira et al., 2020). Segundo dados do Censo da Educação Superior de 2019, houve cerca de 8.603.824 matriculados no 
ensino superior, mas, apenas 1.250 .076 concluintes. Com isto, observa-se que 14,53\% destes, chegam a concluir a graduação (Inep, 2020).

Neste contexto, nota-se que as dificuldades educacionais podem levar ao insucesso estudantil e a evasão, e estas por sua vez, podem ter relação direta ou indireta com a motivação para a aprendizagem e a ausência ou uso inadequado das estratégias e hábitos de estudo (Carvalho, 2012; Porto \& Gonçalves, 2017). Os estudantes que se mostram mais capacitados para as vivências acadêmicas e a formação no ensino superior são aqueles que apresentam hábitos de estudo adequados, que possuem uma boa gestão de tempo associado à organização dos conteúdos e que utilizam de recursos e estratégias de aprendizagem (Matta, 2019).

Esses hábitos se referem ao grau em que o aluno se envolve em práticas regulares de estudo, ao conhecimento e aplicação de métodos de aprendizagem e a capacidade de organização do tempo para atender as demandas das atividades acadêmicas propostas. Credé e Kuncel (2008) afirmam que o estudo eficaz requer não apenas que o aluno possua conhecimento de técnicas e práticas apropriadas, mas também que se tenha autorregulação, capacidade de concentração, esforço contínuo e premeditado, automonitoramento e um senso de responsabilidade e valor na própria aprendizagem.

Contudo, dar-se a oportunidade de perceber como os hábitos de estudo têm uma correlação positiva com o desempenho acadêmico e observar a importância de se reinventar em tempos de crise, buscando estratégias de aprendizagem adaptáveis a cada realidade, a fim de atenuar os desafios dispostos pelo novo cenário educacional. Portanto, este estudo tem por finalidade analisar os hábitos de estudo de acadêmicos de fisioterapia em ensino remoto.

\section{Metodologia}

Trata-se de um estudo transversal, com caráter descritivo, de abordagem quantitativa, realizado com acadêmicos do curso de Fisioterapia de uma Instituição de Ensino Superior (IES) privada, situada no município de Juazeiro do Norte, na região Sul do Ceará, Brasil.

Os estudos transversais visam identificar a frequência em que um dado fenômeno acontece e seus fatores desencadeantes, sendo este, realizado em um curto período, em um determinado momento. Este estudo é adequado para expor características das populações, no que se refere a determinadas variáveis e suas distribuições (Bastos \& Duquia, 2007). Enquanto o caráter descritivo tende a estudar as características de um grupo, levantando opiniões e atitudes de uma determinada população, descobrindo associações entre as variáveis. A abordagem quantitativa, por sua vez, busca a explicação dos fatos e se situa em números, caracterizando-se pelo emprego da quantificação na coleta de informações por meio de técnicas estatísticas (Lira, 2019).

A população do estudo foi composta por estudantes do curso supracitado, de diferentes semestres, com matrícula ativa no período de 2020.1. Na sequência, foi realizado cálculo de população finita, selecionado como amostra um total de 262 acadêmicos, que atendiam aos critérios de elegibilidade.

Foram incluídos na pesquisa todos os estudantes do curso de Fisioterapia, maiores de 18 anos, independente de sexo, que estavam devidamente matriculados, sendo excluídos os acadêmicos que não responderam completamente o questionário utilizado para a coleta de dados, que possuíam acesso parcial a internet e que retiram seu consentimento em algum momento da pesquisa.

O estudo em questão foi realizado em lugares variados em que se encontraram os acadêmicos, já que, em virtude do período de isolamento social diante do enfrentamento a pandemia do novo Coronavírus (COVID-19), ocorreu através de formulário on-line, através do aplicativo de gerenciamento de pesquisa da Google, o Google Forms. O link para acesso ao formulário foi divulgado através de e-mails de turma, aplicativos de conversação e redes sociais, sendo estabelecido o período 
de um mês para acesso (de 13 de agosto a 13 de setembro de 2020). A apreciação dos dados, análise e discussão das informações ocorreu de Agosto a Dezembro de 2020 após aprovação do comitê de ética (CAAE 34661420.7.0000.5048).

Inicialmente, foram coletados dos participantes do estudo informações como idade, sexo, semestre do curso, horas dedicadas ao estudo e presença/ausência de reprovação, para criação de um perfil, além da autopercepção de rendimento. Em seguida, foi utilizado como instrumento, um questionário criado por Ramos et al. (2013) e adaptado pelos presentes pesquisadores, demonstrado no Quadro 1.

Quadro 1 - Questionário "Meus hábitos de estudo".

\begin{tabular}{|c|c|c|c|c|}
\hline \multicolumn{5}{|c|}{ Meus Hábitos de Estudo } \\
\hline $\begin{array}{l}\text { Categoria I - Organização de } \\
\text { estudos }\end{array}$ & Nunca & $\begin{array}{c}\text { As } \\
\text { vezes }\end{array}$ & Frequentemente & Sempre \\
\hline $\begin{array}{l}\text { 1. Tem o hábito de estudar } \\
\text { diariamente? }\end{array}$ & (1) & $(2)$ & (3) & (4) \\
\hline $\begin{array}{l}\text { 2. Tenta organizar as matérias de } \\
\text { estudo de forma sistemática? }\end{array}$ & (1) & (2) & (3) & (4) \\
\hline $\begin{array}{l}\text { 3. Costuma tomar notas e fazer } \\
\text { resumos do conteúdo? }\end{array}$ & (1) & (2) & (3) & (4) \\
\hline $\begin{array}{l}\text { 4. Dedica tempo suficiente para } \\
\text { estudar cada disciplina? }\end{array}$ & (1) & (2) & (3) & (4) \\
\hline $\begin{array}{l}\text { 5. Lê os textos de apoio durante o } \\
\text { período de aula? }\end{array}$ & (1) & (2) & (3) & (4) \\
\hline \multicolumn{5}{|l|}{ Total da Categoria I } \\
\hline $\begin{array}{l}\text { Categoria II - Preparação para } \\
\text { prova }\end{array}$ & Nunca & $\begin{array}{c}\text { As } \\
\text { vezes }\end{array}$ & Frequentemente & Sempre \\
\hline $\begin{array}{l}\text { 6. Estuda as disciplinas muito antes } \\
\text { dos testes ou exames? }\end{array}$ & (1) & $(2)$ & (3) & (4) \\
\hline $\begin{array}{l}\text { 7. Dorme bem na noite anterior a um } \\
\text { teste ou exame? }\end{array}$ & (1) & (2) & (3) & (4) \\
\hline $\begin{array}{l}\text { 8. Quando não entende o conteúdo por } \\
\text { completo procura ajuda? }\end{array}$ & (1) & (2) & (3) & (4) \\
\hline \multicolumn{5}{|l|}{ Total da Categoria II } \\
\hline Categoria III - Local de Estudo & Nunca & $\begin{array}{c}\text { As } \\
\text { vezes }\end{array}$ & Frequentemente & Sempre \\
\hline $\begin{array}{l}\text { 9. O local de estudo é calmo e } \\
\text { silencioso? }\end{array}$ & (1) & $(2)$ & (3) & (4) \\
\hline $\begin{array}{l}\text { 10. O local onde costuma estudar } \\
\text { possui estímulos ou ruídos que podem } \\
\text { o distrair? }\end{array}$ & (1) & (2) & (3) & (4) \\
\hline \multicolumn{5}{|l|}{ Total da Categoria III } \\
\hline $\begin{array}{l}\text { Categoria IV }- \text { Autopercepção } \\
\text { Emocional }\end{array}$ & Nunca & $\begin{array}{c}\text { As } \\
\text { vezes }\end{array}$ & Frequentemente & Sempre \\
\hline $\begin{array}{l}\text { 11. Antes de um teste sente-se } \\
\text { confiante que vai conseguir fazê-lo } \\
\text { com êxito? }\end{array}$ & (1) & $(2)$ & (3) & (4) \\
\hline $\begin{array}{l}\text { 12. Necessita de estímulos para } \\
\text { cumprir os seus deveres? }\end{array}$ & (1) & (2) & (3) & (4) \\
\hline $\begin{array}{l}\text { 13. Desanima facilmente perante uma } \\
\text { tarefa mais complexa? }\end{array}$ & (1) & (2) & (3) & (4) \\
\hline $\begin{array}{l}\text { 14. Procura a colaboração de colegas } \\
\text { mais motivados e responsáveis? }\end{array}$ & (1) & (2) & (3) & (4) \\
\hline $\begin{array}{l}\text { 15. Consegue concentrar-se durante o } \\
\text { estudo? }\end{array}$ & (1) & (2) & (3) & (4) \\
\hline
\end{tabular}

Fonte: Adaptado de Ramos et al. (2011). 
O questionário em questão foi subdividido em quatro categorias e quinze ítens: Organização de estudo (5 ítens); Preparação para prova ( 3 ítens); Local de estudo ( 2 ítens); e Autopercepção emocional (5 ítens). Em cada ítem avaliado, o participante foi incentivado a responder em escala Liket, onde "Nunca" equivalia a 1 ponto, "Às vezes" 2 pontos, "Frequentemente" 3 pontos e, "Sempre", 4 pontos. Ao final, foi realizado um somatório entre as categorias e ao total, havendo a classificação dos hábitos de estudo em: Ruim; Inadequado; Bom; Ótimo, de acordo com a quadro 2.

Quadro 2 - Classificação dos hábitos de estudo de acordo com as categorias.

\begin{tabular}{|l|l|l|l|l|}
\hline & Ruim & Inadequado & Bom & Ótimo \\
\hline Categoria I & De 5 a 9 pontos & De 10 a 14 pontos & De 15 a 19 pontos & 20 pontos \\
\hline Categoria II & De 3 a 5 pontos & De 6 a 8 pontos & De 9 a 11 pontos & 12 pontos \\
\hline Categoria III & De 2 a 3 pontos & De 4 a 5 pontos & De 6 a 7 pontos & 8 pontos \\
\hline Categoria IV & De 5 a 9 pontos & De 10 a 14 pontos & De 15 a 19 pontos & 20 pontos \\
\hline Categorias Totais & De 15 a 29 pontos & De 30 a 44 pontos & De 45 a 59 pontos & 60 pontos \\
\hline
\end{tabular}

Fonte: Lemos et al. (2021).

Após a obtenção dos dados da pesquisa, esses foram transportados para os programas Microsoft Office Excel® 2010 e, posteriormente, exportados para o GraphPad Prism e Stata Softwere para análise, onde foram realizados os testes de correlações de Pearson e Spearman sendo aceito o índice de significância de $P>0,05$.

\section{Resultados}

Participaram desta pesquisa 262 acadêmicos de fisioterapia, matriculados na instituição pesquisada, no período da coleta de dados. Os participantes eram, em sua maioria do sexo feminino (76,72\%), com idade média de 20,16 anos de idade e pertencentes aos últimos anos de curso (54,58\%), como é possível observar na Tabela 1. Quanto as características educacionais, a maior parcela relata nunca ter reprovado disciplinas no decorrer da graduação $(61,83 \%)$, além de afirmar estudar remotamente entre uma e três horas por dia $(37,79 \%)$. 
Research, Society and Development, v. 11, n. 1, e59111125433, 2022

(CC BY 4.0) | ISSN 2525-3409 | DOI: http://dx.doi.org/10.33448/rsd-v11i1.25433

Tabela 1 - Caracterização da amostra participante da pesquisa.

\begin{tabular}{lll}
\hline Variável & n (262) & \% \\
\hline Sexo & & \\
Feminino & 201 & $76,72 \%$ \\
Masculino & 61 & $23,28 \%$ \\
Idade & & \\
De 18 a 24 anos & 215 & $82,06 \%$ \\
De 25 a 30 anos & 35 & $13,36 \%$ \\
Acima de 30 anos & 12 & $4,58 \%$ \\
Semestre & & \\
$1^{\circ}$ ao $4^{\circ}$ semestre & 67 & $25,57 \%$ \\
$5^{\circ}$ ao $7^{\circ}$ semestre & 52 & $19,85 \%$ \\
$8^{\circ}$ ao $10^{\circ}$ semestre & 143 & $54,58 \%$ \\
Reprovação & & \\
Não & 162 & $61,83 \%$ \\
Sim & 100 & $38,17 \%$ \\
Horas de Estudo Remoto & & \\
Até 1 hs/dia & 86 & $32,82 \%$ \\
Entre 1 e 3 hs/dia & 99 & $37,79 \%$ \\
De 3 a 5 hs/dia & 55 & $20,99 \%$ \\
Acima de 5 hs/dia & 22 & $8,40 \%$ \\
\hline
\end{tabular}

Fonte: Lemos et al. (2021).

Ao analisarmos os hábitos dos acadêmicos em ensino remoto, estratificados nas 4 categorias, percebe-se a prevalência de hábitos inadequados em todas elas, com maiores percentuais na Categoria III - Local de Estudo (74,05\%) e na Categoria IV - Autopercepção Emocional (74,81\%). Na análise do somatório geral, observa-se percentuais ainda maiores classificados como inadequados $(83,59 \%)$. Todos os dados podem ser observados na Tabela 2. 
Research, Society and Development, v. 11, n. 1, e59111125433, 2022

(CC BY 4.0) | ISSN 2525-3409 | DOI: http://dx.doi.org/10.33448/rsd-v11i1.25433

Tabela 2 - Número absolutos e percentuais da classificação dos hábitos remotos.

\begin{tabular}{|c|c|c|c|c|c|c|}
\hline Classificação & n (262) & $\%$ & Min. & Máx. & Média & $\begin{array}{l}\text { Desvio } \\
\text { Padrão }\end{array}$ \\
\hline Categoria I & & & 6 & 20 & 13,03 & 2,845 \\
\hline Ruim & 23 & $8,78 \%$ & & & & \\
\hline Inadequado & 156 & $59,54 \%$ & & & & \\
\hline Bom & 78 & $29,77 \%$ & & & & \\
\hline Ótimo & 5 & $1,91 \%$ & & & & \\
\hline Categoria II & & & 3 & 12 & 7,62 & 1,741 \\
\hline Ruim & 28 & $10,69 \%$ & & & & \\
\hline Inadequado & 164 & $62,60 \%$ & & & & \\
\hline Bom & 62 & $23,66 \%$ & & & & \\
\hline Ótimo & 8 & $3,05 \%$ & & & & \\
\hline Categoria III & & & 2 & 7 & 4,88 & 0,852 \\
\hline Ruim & 9 & $3,44 \%$ & & & & \\
\hline Inadequado & 194 & $74,05 \%$ & & & & \\
\hline Bom & 59 & $22,52 \%$ & & & & \\
\hline Ótimo & 0 & $0,00 \%$ & & & & \\
\hline Categoria IV & & & 7 & 20 & 12,16 & 2,163 \\
\hline Ruim & 24 & $9,16 \%$ & & & & \\
\hline Inadequado & 196 & $74,81 \%$ & & & & \\
\hline Bom & 41 & $15,65 \%$ & & & & \\
\hline Ótimo & 1 & $0,38 \%$ & & & & \\
\hline Total das Categorias & & & 23 & 57 & 37,7 & 5,577 \\
\hline Ruim & 16 & $6,11 \%$ & & & & \\
\hline Inadequado & 219 & $83,59 \%$ & & & & \\
\hline Bom & 27 & $10,31 \%$ & & & & \\
\hline Ótimo & 0 & $0,00 \%$ & & & & \\
\hline
\end{tabular}

Fonte: Lemos et al. (2021).

Ao analisarmos a autopercepção de rendimento no ensino remoto, relacionando este com a classificação geral dos hábitos de estudo, percebe-se que os maiores percentuais de hábitos de estudo ruins ou inadequados são notados em indivíduos que avaliam seu rendimento como péssimo $(18,18 \%$ e $72,73 \%)$ ou ruim $(11,11 \%$ e $81,48 \%)$. Contudo, o hábito de estudo inadequado é predominante em todas as percepções de rendimento, como é possível ver no Gráfico 1. 
Gráfico 1 - Relação entre a autopercepção de rendimento no ensino remoto e a classificação geral dos hábitos de estudo dos indivíduos avaliados.

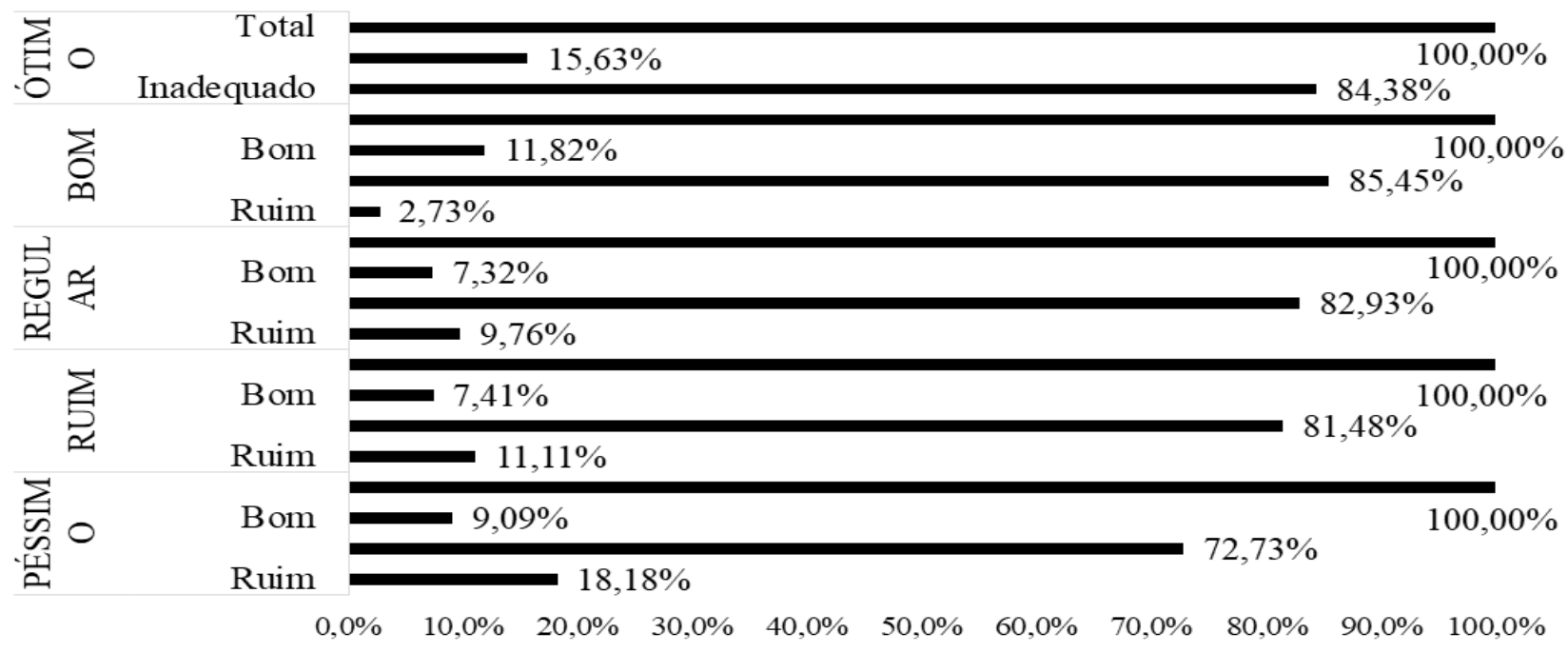

Fonte: Lemos et al. (2021).

Para determinação de correlação entre os valores atribuídos para a importância do ensino remoto e os valores de somatório de cada categoria de hábitos de estudo, assim como a classificação geral, foram realizados os testes de Correlações de Pearson e Spearman, não sendo identificado em ambas quaisquer correlações, como mostra a tabela 3.

Tabela 3 - Correlações de Pearson e Spearman entre a "Importância atribuída ao ensino remoto" e os somatórios das categorias do Questionário dos Hábitos de Estudo.

\begin{tabular}{lll}
\hline Importância atribuída ao ensino remoto frente à: & Pearson (r) & Spearman (r) \\
\hline Organização do estudo (C1) & 0,2096 & 0,2878 \\
Preparação para prova (C2) & 0,1827 & 0,2100 \\
Local de estudo (C3) & 0,1238 & 0,0820 \\
Autopercepção emocional (C4) & 0,1044 & 0,1351 \\
Hábitos de estudo remoto (C Total) & 0,2234 & 0,2740 \\
\hline
\end{tabular}

Fonte: Lemos et al. (2021).

\section{Discussão}

No presente estudo foi possível conhecer melhor o perfil de acadêmicos de Fisioterapia e identificar os hábitos de estudo no ensino remoto. Em relação ao gênero, a predominância de participantes era do sexo feminino (76,72\%), achado semelhante ao perfil montado no estudo de Guimarães et al. (2017) sobre estilo de vida e fatores associado entre estudantes universitários, no qual a predominância foi de $66,2 \%$ do gênero feminino. Os autores em questão associam essa porcentagem ao fato da grande parte da sociedade ser constituída por mulheres. Além disso, a literatura ainda comprova que as mulheres são maioria no ensino superior e esse é o público mais frequente nas pesquisas que envolvem universitários (Alves et al., 2017; Valles et al., 2018; Ferraz et al., 2019).

Em relação ao quesito idade, a prevalência maior foi dos acadêmicos de 18 a 24 anos, faixa etária que corresponde ao período no qual as pessoas ingressam na universidade (Mendes et al., 2016). Além disso, também foi observado que estes, em sua maioria, relataram já ter reprovado alguma disciplina durante a graduação. Meurer et al. (2018), compara em sua pesquisa o rendimento acadêmico dos discentes que reprovaram em pelo menos alguma disciplina com os demais estudantes, mostrando 
através do Teste de Mann-Whitney a comprovação por meio de estatísticas de que os alunos reprovados possuem coeficiente de rendimento menor, apresentando diferença de 0,1358 em relação ao grupo dos não reprovados, mesmo desconsiderando a disciplina reprovada, pois apresenta propensão de um menor desempenho não só dessa matéria como também nas demais.

Quanto aos hábitos de estudo e rendimento em aulas, existem variados aspectos que são considerados influentes no grau de rendimento do aluno, um deles é o tempo dedicado ao estudo fora da sala de aula como aspecto complementar do processo ensino/aprendizagem. As horas de estudo extraclasses semanais influenciam 36\% no rendimento acadêmico dos alunos (Gonçalves et al., 2015). Nos resultados do estudo em questão, os estudantes afirmam estudar remotamente entre uma e três horas por dia. Achado similar ao da pesquisa realizada por Fernandes et al. (2020) com discentes do curso de Fisioterapia da UniEvangélica, que relataram ter o hábito de estudar 15hs semanais, o que equivale a uma média 2, 14 horas por dia.

Já no estudo de Noro e Moya (2019), os autores mostram o efeito das horas de estudo em avaliações além da universidade, sinalizando que os alunos que dedicaram oito ou mais horas de estudo por semana, conseguiram obter um bom desempenho nos exames. Além de uma boa gestão do tempo, faz-se necessário que os alunos possuam estratégias de aprendizagem para obter um bom desempenho acadêmico, tais quais: estratégias de auto-avaliação, organização e transformação, estabelecimento de metas e planeamento, busca de informação, registro de informação, auto-monitorização, organização do ambiente, busca de ajuda e revisão (Casiraghi, 2020).

Os resultados da presente pesquisa evidenciam que os discentes apresentaram-se com hábitos de estudo inadequados quanto à organização do estudo $(59,54 \%)$, preparação para prova $(62,60 \%)$, local de estudo $(74,05 \%)$ e auto percepção emocional (74,81\%), dados que podem estar associados à mudança sofrida no processo educacional, que ao funcionar de forma remota, se depara com questões que precisam ser consideradas, como o acesso à internet, equipamentos apropriados para o estudo, a realidade socioeconômica de cada família e condições psicológicas do sujeito, visto que estes fatores interferem diretamente na forma como o aluno vai lidar com o processo de aprendizagem e hábitos de estudo (Teles et al., 2020).

Os achados descritos sobre os hábitos inadequados em todas as categorias, no presente estudo, podem ter relação aos achados no estudo apresentado por Silva et al. (2020). Neste os autores trazem o resultado de um questionamento realizado com discentes sobre os desafios do ensino remoto emergencial, sendo apontada pelos entrevistados a dificuldade em concentrar-se, representando $63 \%$ dos apontamentos, a falta de um espaço favorável para estudo em suas residências, que está diretamente ligada com a questão da dispersão da atenção, e a falta de planejamento e preparo das atividades para alcançar suas metas de aprendizagem.

Diferente do que já foi exposto, o estudo de Barreto et al. (2019), com alunos do curso de Medicina, investigou as estratégias utilizadas no estudo individual e a percepção em relação à aprendizagem, apresentando hábitos de estudo satisfatório associados à uma boa organização e planejamento da aprendizagem, utilizando de recursos apropriados. A maioria dos alunos da pesquisa em questão dispunha de recursos eletrônicos para acessar a internet, julgam fácil cumprir o horário de estudo e afirmam evitar situações que desviam sua atenção. Grande parte relata elaborar esquemas e resumos e utilizar mais de um livro-texto em seus estudos, acessam a artigos por meio de plataformas de bases de dados científicos e fazem uso de apostilas comerciais e resumos não próprios.

Corroborando com o trabalho supracitado Matta (2019), ressalta que os estudantes que possuem bons hábitos de estudo com uma boa gestão do tempo, organização do conteúdo, utilização de recursos e estratégias de aprendizagem e que se preparam corretamente para as avaliações, se mostraram mais capacitados para as vivências acadêmicas e a formação no ensino superior. O rendimento acadêmico é explicado pela capacidade de se envolver, planejar e cumprir prazos em relação às atividades propostas e pela percepção de bons hábitos de estudo. Ao investigar a autopercepção de rendimento dos entrevistados deste estudo no ensino remoto, verifica-se que os maiores percentuais de hábitos de estudo ruins ou inadequados são notados em indivíduos que avaliam seu rendimento como péssimo ou ruim. 
Os estudantes com autopercepção de desempenho acadêmico alta detêm mais características como persistência e satisfação por se sentirem desafiados, se apresentando com maior potencial para autorregularem a sua aprendizagem do que aqueles com autopercepção de desempenho baixa, que por sua vez, possuem característica de evitação. Possivelmente, a autoavaliação negativa dar-se pela sensação de inferioridade (Dalbosco et al., 2018). Indo em convergência, Ariño e Bardagi (2018) salientam que possuir uma percepção positiva acerca das próprias habilidades e competências para aprender, empregar estratégias de estudo e gerir o tempo, pode se constituir como fator protetivo para saúde mental do indivíduo.

\section{Considerações Finais}

Conclui-se, portanto, que o perfil de acadêmicos avaliados, caracterizado por predomínio do gênero feminino, com média de idade de 20,16 anos, pertencem aos últimos anos do curso de Fisioterapia. A maioria relata nunca ter reprovado disciplinas no decorrer da graduação e afirmam estudar remotamente entre uma e três horas por dia. Os estudantes apresentaram-se com hábitos de estudo inadequados no ensino remoto em todas as categorias investigadas e, ao analisar a autopercepção de rendimento nessa modalidade de ensino, o hábito inadequado é predominante em todas as percepções de rendimento acadêmico.

O conhecimento do perfil discente, aliado às suas percepções acadêmicas e hábitos de estudo, podem conferir adaptações necessárias à continuidade do processo ensino-aprendizagem no ensino remoto. Destarte, sugere-se haver novas pesquisas acerca desta temática, pois entender os aspectos que influenciam o desempenho dos alunos é de grande importância no contexto atual da educação, fundamentada nas informações que isso pode proporcionar, sendo úteis no processo decisório de determinação de políticas que visem melhorar a qualidade de ensino nas instituições, visto que as universidades detêm um papel primordial dentro da construção e formação social dos estudantes.

\section{Referências}

Alves, E. V. D. S. B., dos Santos, M. A., da Ponte, A. V. A., da Silva Rosa, J. L., Faria, A. C. D., Manoel, F., \& Braga, J. C. (2017). Autopercepção de habilidades sociais e adaptação à vida acadêmica. Cadernos de educação, saúde e fisioterapia, 4(8).

Ariño, D. O., \& Bardagi, M. P. (2018). Relação entre fatores acadêmicos e a saúde mental de estudantes universitários. Revista psicologia em pesquisa, 12(3).

Barreto, I. D. P., Gomes, P. A., Furlaneto, I. P., \& Barreto, B. (2019). Avaliação das estratégias de autoaprendizagem em alunos de um curso de medicina em Belém-Pará. Revista Brasileira de Educação Médica, 43, 36-46.

Bastos, J. L. D., \& Duquia, R. P. (2007). Um dos delineamentos mais empregados em epidemiologia: estudo transversal. Scientia Medica, 17(4), 229-232.

Casiraghi, B., Boruchovitch, E., \& Almeida, L. S. (2020). Crenças de autoeficácia, estratégias de aprendizagem e o sucesso acadêmico no Ensino Superior. Revista E-Psi-Revista Portuguesa de Acesso Livre que publica.

Carvalho, P. D. S. (2012). Hábitos de estudo e sua influência no rendimento escolar (Doctoral dissertation, [sn]).

Cavalcante, A. S. P., Machado, L. D. S., Farias, Q. L. T., Pereira, W. M. G., \& da Silva, M. R. F. (2020). Educação superior em saúde: a educação a distância em meio à crise do novo coronavírus no Brasil. Avances en Enfermería, $38(1 \mathrm{supl})$.

Credé, M., \& Kuncel, N. R (2008). Hábitos de estudo, habilidades e atitudes: o terceiro pilar que sustenta o desempenho acadêmico universitário. Perspectives on psicológico science 3 (6), 425-453.

Dalbosco, S. N. P., Ferraz, A. S., \& dos Santos, A. A. A. (2018). Metas de realização, autorregulação da aprendizagem e autopercepção de desempenho em universitários. Revista Brasileira de Orientação Profissional, 19(1), 75-84.

Fernandes, V. L. S., Melo, C. M., Alves, D. C., Souza, F. B., Zani, H. P., Nishi, M., \& Fontoura, H. S. (2020). A percepção dos discentes do curso de fisioterapia frente ao ensino remoto durante a pandemia. Anais do Seminário de Atualização de Práticas Docentes, 2(2).

Ferraz, A. S., dos Santos, A. A. A., \& Almeida, L. S. (2019). Escala de Atribuições de Causalidade e Rendimento Escolar: Estudo de Evidência de Validade de Critério. Revista Portuguesa de Educação, 32(1), 125-139.

Fior, Camila Alves, \& Mercuri, Elizabeth. (2018). Envolvimento acadêmico no ensino superior e características do estudante. Revista Brasileira de Orientação Profissional, 19(1), 85-95

Garcia, T. C. M., Morais, I. R. D., Zaros, L. G., \& Rêgo, M. C. F. D. (2020). Ensino remoto emergencial: proposta de design para organização de aulas. Natal: SEDIS/UNFR, p.1-18. 
Research, Society and Development, v. 11, n. 1, e59111125433, 2022

(CC BY 4.0) | ISSN 2525-3409 | DOI: http://dx.doi.org/10.33448/rsd-v11i1.25433

Gonçalves, M. P. G.; et al. (2015). Influência do tempo de estudo no rendimento do aluno universitário. Revista Fundamentos, 2(2), 1-17.

Guimarães, M. R., Batista, A. M. D. O., Santos, I. M. D. S., Vale, M. D. P. S. S., Moura, I. H. D., \& Silva, A. R. V. D. (2017). Estilo de vida e fatores associados entre estudantes universitários. Rev. enferm. UFPE on line, 3228-3235.

Inep (2020). Censo da Educação Superior 2019: divulgação dos resultados. Ministério da Educação, Brasília.

Lira, B. C. (2019). Passo a passo do trabalho científico. Editora Vozes.

Matta, C. M. B. D. (2019). Influência das vivências acadêmicas e da autoeficácia na adaptação, rendimento e evasão de estudantes nos cursos de engenharia de uma instituição privada. Tese (Doutorado em Psicologia da Saúde) - Psicologia, Universidade Metodista de São Paulo, São Paulo, p. 1-163.

Mendes, M. L. M., da Silva, F. R., de Omena Messia, C. M. B., de Carvalho, P. G. S., \& da Silva, T. F. A. (2016). Hábitos alimentares e atividade física de universitários da área de saúde do município de Petrolina-PE. Tempus Actas de Saúde Coletiva, 10(2), ág-205.

Meurer, A. M., Pedersini, D. R., Antonelli, R. A., \& Voese, S. B. (2018). Estilos de aprendizagem e rendimento acadêmico na universidade. REICE: Revista Iberoamericana sobre Calidad, Eficacia y Cambio en Educación, 16(4), 23-43.

Noro, L. R. A., \& Moya, J. L. M. (2019). Condições sociais, escolarização e hábitos de estudo no desempenho acadêmico de concluintes da área da saúde. Trabalho, Educação e Saúde, 17.

Nunes, RCA e Dutra, CM (2020). Ensino remoto para alunos do Atendimento Educacional Especializado. Research, Society and Development, 9 (11), e64291110060-e64291110060.

Oliveira, T. M., de Lima Santos, F. A., de Lima, W. P., Bezerra, M. A. A., dos Santos Camurça, A. J., de Lavor Farias, E., ... \& Neto, I. C. P. (2020). Percepção de acadêmicos sobre a relação entre a participação em grupos de estudo e rendimento em avaliações. Research, Society and Development, 9(12), e15091211027-e15091211027.

Porto, R. C., \& Gonçalves, M. P. (2017). Motivação e envolvimento acadêmico: um estudo com estudantes universitários. Psicologia Escolar e Educacional, $21,515-522$.

Ramos, A. L. M., Nogueira, A. D. B. L., Ferraz, D. P. D. A., Brezolin, L. M. T. D. F., Pinto, L. M., \& Muniz, W. D. F. (2011). Questionário de Hábitos de Estudo para estudantes universitários: validação e precisão. Paidéia (Ribeirão Preto), 21, 363-371.

Silva, A. C. O., de Araújo Sousa, S., \& de Menezes, J. B. F. (2020). O ensino remoto na percepção discente: desafios e benefícios. Dialogia, (36), 298-315.

Teles, E. C., Campana, A., Costa, S., \& Nascimento, F. (2020). O ensino remoto e os impactos nas aprendizagens. Revista ComSertões, 9(2), 72-90.

Valles, I. A., Facio-Arciniega, S., Quiroz-Guerra, A., Alemán-de la Torre, L., Flores-Ramírez, M., \& Rosales-González, M. (2018). Atitude, hábitos de estudo e rendimento académico: Abordagem desde a teoria da ação racional. Enfermería universitaria, 15(4), 342-351. 